\title{
ULTRASONIC WAVES IN MAGNETIC LIQUIDS: TWO-PHASE APPROACH AND EXPERIMENTAL RESULTS
}

\author{
M. KACZMAReK ${ }^{a}$, T. HoRnowski ${ }^{b}$, A. SKUMIEL ${ }^{b}$ AND M. EABOWSKI ${ }^{b}$ \\ ${ }^{a}$ Department of Environmental Mechanics, Pedagogical University in Bydgoszcz \\ Chodkiewicza 30, 85-064 Bydgoszcz, Poland \\ ${ }^{b}$ Institute of Acoustics, Adam Mickiewicz University \\ Umultowska 85, 61-614 Poznań, Poland
}

(Received September 6, 1999; revised version November 29, 1999; in final form February 2, 2000)

A two-phase model of dynamical behavior of magnetic liquid influenced by constant magnetic field is considered. The model takes into account mutual interaction of clusters giving skeleton's stiffness and interaction of clusters with free liquid producing attenuation and dispersion of waves for transversally isotropic system. Rotational degrees of freedom are disregarded. Predictions of the model are discussed for variable amount of clusters and compared with experimental data for different magnetic fields.

PACS numbers: $47.35 .+\mathrm{i}, 75.50 .-\mathrm{y}, 62.60 .+\mathrm{v}$

\section{Introduction}

In the past few decades an increase in technological importance as well as significant interest in theoretical and experimental investigations of magnetic liquids are observed. Magnetic liquids when not subjected to magnetic field are homogeneous colloidal suspensions of ferromagnetic particles in carrier liquids, such as water, oil or kerosene. Typically, the diameters of solid particles range from 5 to 10 nanometers. To prevent coagulation of the particles they are coated with surface-active dispersive medium. However, experimental investigations of magnetic liquids in DC magnetic field show that a certain amount of colloidal particles forms aggregates (clusters) which tend to join into a chain-like structure as long as hundreds of nanometers [1]. This gel-like microstructure causes an increase in stiffness of the liquid, induces anisotropy of its mechanical properties, and contributes to the dissipation of energy of internal processes due to interaction of clusters with the surrounding liquid.

A useful way to study properties of magnetic liquids is based on application of ultrasonic methods. These methods proved to be very powerful to observe anisotropy of mechanical properties of the liquids in variable magnetic field and 
a wide range of temperature. They can be also used to study the influence of an internal structure of magnetic liquid constituted by clusters on macroscopic rheological characteristics of the liquids.

Numerous models of ultrasonic wave propagation in magnetic liquids have been proposed, see e.g. [2-6]. They are based on magnetohydrodynamics, theory of liquid crystals or theory of mixtures. However, most of them fail to predict properly some of the observed features of the liquids such as anisotropy of attenuation and phase velocity of ultrasonic waves, and dependence of wave propagation parameters on strength of magnetic field and temperature. The promising model which offers a description of characteristic features of attenuation of mechanical waves is given by Taketomi [4]. This model takes into account the presence and motion of clusters in magnetic liquid and in particular the mutual interaction between different clusters and the interaction between clusters and free fluid. Since the Taketomi approach to treat the magnetic liquid as a mixture is based solely on an analysis of dissipation of energy of the system it is limited to the study of attenuation and in fact cannot predict dispersion of waves as well as other consequences of the two-phase nature of magnetic liquid (e.g. the total number of wave modes in the liquid that can propagate taking into account a given anisotropy and stiffness of skeleton).

A more thorough two-phase approach founded on field equations for mass and momentum of two-component mixture was applied for the description of ultrasonic waves in magnetic liquids by Lipkin [5], Gogosov et al. [6], and Bădescu et al. [7]. This model, however, treats the magnetic liquid as a suspension in which interactions between clusters and as a result the existence of a skeleton composed of clusters having certain stiffness and anisotropy are neglected.

The purpose of the present paper is to develop a linear two-phase model of wave propagation in magnetic liquid using the concept of superimposed continua. The governing equations of the model are formulated using the theory of multiphase media, and particularly the theory of anisotropic fluid saturated porous materials, e.g. [8, 9], and [10]. It is assumed that the two phases of the medium are: the skeleton made of interacting chains of clusters and the liquid composed of carrier liquid with free colloidal particles (particles not gathered into clusters). The mutual interactions of clusters are represented through components of stress tensor for the skeleton. The interactions of clusters with liquid are expressed as the sum of viscous and inertial drag forces. Given the assumed transversal isotropy of the medium induced by DC magnetic field the possible wave modes predicted by the model in infinite medium are specified. Quantitative results obtained from the model for a fast quasi-longitudinal wave and variable amount of interacting clusters are discussed. The numerical predictions are compared with experimental data.

\section{Model description}

Following the introductory assumptions the magnetic liquid is modeled as consisting of two phases: solid skeleton (s) made of interacting clusters and fluid (f) composed of carrier liquid with free magnetic particles. The motion of the phases 
is governed by the macroscopic linearized balances of linear momentum

$$
\rho^{\mathrm{f}} \frac{\partial v^{\mathrm{f}}}{\partial t}-\nabla \cdot \boldsymbol{T}^{\mathrm{f}}-\boldsymbol{R}^{\mathrm{f}}=0, \quad \rho^{\mathrm{s}} \frac{\partial v^{\mathrm{s}}}{\partial t}-\nabla \cdot \boldsymbol{T}^{\mathrm{s}}-\boldsymbol{R}^{\mathrm{s}}=0,
$$

where $\rho^{f}$ and $\rho^{s}$ stand for the average mass densities of fluid and solid phase, respectively, $v^{\mathrm{f}}$ and $v^{\mathbf{s}}$ are macroscopic velocities of the fluid and solid skeleton, respectively, $\boldsymbol{T}^{\mathrm{f}}$ and $\boldsymbol{T}^{\mathrm{s}}$ denote stress tensors, and $\boldsymbol{R}^{\mathrm{f}}=-\boldsymbol{R}^{\mathbf{s}}$ are interaction forces between the phases. All the above quantities are understood as averages over volume regimes which are much smaller than the wavelength of the considered waves.

The proposed model neglects rotational degrees of freedom of magnetic particles, the momentum interaction of the particles with carrier. liquid and external magnetic field and the mutual momentum interaction of the particles (antisymmetry of the stress tensor). This assumption is justified if the direction of magnetic field is parallel to the propagation vector of ultrasonic wave.

The stress tensors and interaction forces are assumed to be constitutive functions that express material properties of the medium. The stress tensor in fluid phase is split into two parts: hydrostatic and viscous components

$$
T_{i j}^{\mathrm{f}}=-(1-n) p \delta_{i j}+\pi_{i j}
$$

where $p$ is the average of microscopic hydrostatic pressure, $n$ is the volume fraction of clusters which constitute the skeleton and $\pi_{i j}$ is the macroscopic viscous stress tensor. Assuming that axis $x_{3}$ is perpendicular to the isotropy plane which includes axes $x_{1}$ and $x_{2}$, the hydrostatic component of the stress tensor in fluid phase for the transversally isotropic medium reads [9]

$$
-(1-n) p=M\left(e_{11}+e_{22}\right)+Q e_{33}+R \epsilon,
$$

where $e_{i j}$ denotes the strain tensor for the skeleton and $\epsilon$ is the relative volume change (dilatation) of fluid that can be expressed by displacements of skeleton, $u_{i}^{s}$, and fluid, $u_{i}^{\mathrm{f}}$, as following:

$$
e_{i j}=\frac{1}{2}\left(\frac{\partial u_{i}^{\mathbf{s}}}{\partial x_{j}}+\frac{\partial u_{j}^{\mathbf{s}}}{\partial x_{i}}\right)
$$

and

$$
\epsilon=\frac{\partial u_{i}^{\mathrm{f}}}{\partial x_{i}}
$$

The coefficients $M$ and $Q$ relate deformations of solid to that of fluid while $R$ is a measure of the hydrostatic stress required to change the volume of fluid phase if the volume of skeleton remains constant. The components of the macroscopic viscous stress tensor in fluid phase are assumed to be dependent on rate of deformation of fluid

$$
\boldsymbol{d}_{i j}=\frac{1}{2}\left(\frac{\partial v_{i}^{\mathrm{f}}}{\partial x_{j}}+\frac{\partial v_{j}^{\mathrm{f}}}{\partial x_{i}}\right)
$$

and for a given symmetry are the following:

$$
\begin{aligned}
& \pi_{11}=2 \mu d_{11}+\alpha\left(d_{11}+d_{22}+d_{33}\right), \\
& \pi_{22}=2 \mu d_{22}+\alpha\left(d_{11}+d_{22}+d_{33}\right),
\end{aligned}
$$




$$
\begin{aligned}
& \pi_{33}=2 \gamma d_{33}+\beta\left(d_{11}+d_{22}+d_{33}\right), \\
& \pi_{13}=\pi_{31}=2 \delta d_{13}, \\
& \pi_{23}=\pi_{32}=2 \delta d_{23} \\
& \pi_{12}=\pi_{21}=2 \mu d_{12}
\end{aligned}
$$

where $\mu, \alpha, \gamma, \beta$, and $\delta$ are the viscosity constants.

The stress tensor for the skeleton expressing interaction between clusters depends in general on deformation of skeleton and dilatation of fluid and its components for transversally isotropic case take on the following form [9]:

$$
\begin{aligned}
& T_{11}^{\mathrm{s}}=2 N e_{11}+A\left(e_{11}+e_{22}\right)+F e_{33}+M \epsilon, \\
& T_{22}^{\mathrm{s}}=2 N e_{22}+A\left(e_{11}+e_{22}\right)+F e_{33}+M \epsilon, \\
& T_{33}^{\mathrm{s}}=2 C e_{33}+F\left(e_{11}+e_{22}\right)+Q \epsilon, \\
& T_{13}^{\mathrm{s}}=T_{31}^{\mathrm{s}}=2 L e_{13}, \\
& T_{23}^{\mathrm{s}}=T_{32}^{\mathrm{s}}=2 L e_{23}, \\
& T_{12}^{\mathrm{s}}=T_{21}^{\mathrm{s}}=2 N e_{12},
\end{aligned}
$$

where $N, A, F, C$, and $L$ are the constants characterizing elastic response of the skeleton.

The interaction force between carrier liquid and the skeleton is assumed to be a sum of viscous (frictional) force and inertial force and the appropriate components are the following:

$$
\begin{aligned}
& R_{1}^{\mathrm{s}}=-b_{t}\left(v_{1}^{\mathrm{f}}-v_{1}^{\mathrm{s}}\right)-c_{t} \frac{\partial}{\partial t}\left(v_{1}^{\mathrm{f}}-v_{1}^{\mathrm{s}}\right), \\
& R_{2}^{\mathrm{s}}=-b_{t}\left(v_{2}^{\mathrm{f}}-v_{2}^{\mathrm{s}}\right)-c_{t} \frac{\partial}{\partial t}\left(v_{2}^{\mathrm{f}}-v_{2}^{\mathrm{s}}\right), \\
& R_{3}^{\mathrm{s}}=-b_{n}\left(v_{3}^{\mathrm{f}}-v_{3}^{\mathrm{s}}\right)-c_{n} \frac{\partial}{\partial t}\left(v_{3}^{\mathrm{f}}-v_{3}^{\mathrm{s}}\right),
\end{aligned}
$$

where $b_{t}, b_{n}, c_{t}$, and $c_{n}$ are the coefficients characterizing viscous and inertial couplings in a transversally isotropic medium. Taking into account the fact that in this paper the waves in ultrasonic frequency range are considered, the above specified interaction force does not include the component which represents the history dependence of the interaction between phases (Basset force) $[6,10]$.

Combining the above introduced constitutive functions and equations of linear momentum for solid and fluid phase one can obtain the equations for macroscopic displacements of phases. In particular, the equations govern the propagation of plane harmonic waves in an infinite medium for which the solutions take on the following form: $u_{i}^{\mathrm{f}}=\bar{u}_{i}^{\mathrm{f}} \exp \left[\mathrm{i} \omega\left(t-q_{j} x_{j}\right)\right]$, and $u_{i}^{\mathrm{s}}=\bar{u}_{i}^{\mathrm{s}} \exp \left[\mathrm{i} \omega\left(t-q_{j} x_{j}\right)\right]$, where $\bar{u}^{\mathrm{f}}=\left\{\bar{u}_{1}^{\mathrm{f}}, \bar{u}_{2}^{\mathrm{f}}, \bar{u}_{3}^{\mathrm{f}}\right\}$ and $\bar{u}^{\mathrm{s}}=\left\{\bar{u}_{1}^{\mathrm{s}}, \bar{u}_{2}^{\mathrm{s}}, \bar{u}_{3}^{\mathrm{s}}\right\}$ are amplitudes of displacement of fluid and solid, $\omega$ is the angular frequency, and $q=\left\{q_{1}, 0, q_{3}\right\}$ is the slowness vector which is related to the complex wave vector, $k$, by the relation $k=\omega q$. The components of the slowness vector are chosen in such way that the plane of constant phase is parallel to the axis $x_{2}$. Plugging the solutions into the governing equations 
of the two-phase model of magnetic liquid one obtains six homogeneous equations with unknown amplitudes $\bar{u}_{i}^{\mathrm{f}}$ and $\bar{u}_{i}^{\mathrm{s}}$

$$
\begin{aligned}
& D_{11} \bar{u}_{1}^{\mathrm{s}}+D_{12} \bar{u}_{3}^{\mathrm{s}}+D_{13} \bar{u}_{1}^{\mathrm{f}}+D_{14} \bar{u}_{3}^{\mathrm{f}}=0, \\
& D_{31} \bar{u}_{1}^{\mathrm{s}}+D_{32} \bar{u}_{3}^{\mathrm{s}}+D_{33} \bar{u}_{1}^{\mathrm{f}}+D_{34} \bar{u}_{3}^{\mathrm{f}}=0, \\
& D_{41} \bar{u}_{1}^{\mathrm{s}}+D_{12} \bar{u}_{3}^{\mathrm{s}}+D_{13} \bar{u}_{1}^{\mathrm{f}}+D_{14} \bar{u}_{3}^{\mathrm{f}}=0, \\
& D_{61} \bar{u}_{1}^{\mathrm{s}}+D_{62} \bar{u}_{3}^{\mathrm{s}}+D_{63} \bar{u}_{1}^{\mathrm{f}}+D_{64} \bar{u}_{3}^{\mathrm{f}}=0, \\
& D_{21} \bar{u}_{2}^{\mathrm{s}}+D_{22} \bar{u}_{2}^{\mathrm{f}}=0, \\
& D_{51} \bar{u}_{2}^{\mathrm{s}}+D_{52} \bar{u}_{2}^{\mathrm{f}}=0,
\end{aligned}
$$

where the coefficients $D_{i j}$ are the combinations of the material constants, angular frequency, and components of the slowness vector for which the explicit forms are given in Appendix. Since only the last two equations contain amplitudes $\bar{u}_{2}^{\mathrm{f}}$ and $\bar{u}_{2}^{\mathrm{s}}$, the equations are independent of the other equations, and one can notice that the waves which are related to the motion of particles in $x_{2}$-direction are not coupled with the waves with the motion of particles in the plane determined by $x_{1}$ and $x_{3}$. The set of four homogeneous Eqs. $(22-25)$ has a non-trivial solution if the appropriate determinant of the matrix of coefficients, $D^{\mathrm{I}}$, is equal to zero, $D^{\mathrm{I}}=0$. This condition yields a dispersion equation corresponding to the waves which are disturbances of displacement in the plane of $x_{1}$ and $x_{3}$. Similarly, the uniqueness of the solution of Eqs. (26) and (27) demands that the corresponding determinant is equal to zero, $\operatorname{det} \boldsymbol{D}^{\mathrm{II}}=0$. This condition yields a dispersion equation corresponding to the propagation of the waves with a motion of particles in direction of $x_{2}$.

Introducing into the dispersion equations a complex slowness parameter $\bar{\alpha}$ such that $q_{1}=\bar{\alpha} \sin \theta$, and $q_{3}=\bar{\alpha} \cos \theta$, where $\theta$ is the angle between the magnetic field and the direction of wave propagation, one obtains the dispersion equations in forms of polynomials in $\bar{\alpha}$. The roots of the polynomials with positive values of real components of $\bar{\alpha}$ enable us to determine the number of the wave modes predicted by the model as well as the velocities and the coefficients of attenuation of the waves. The model predicts four types of waves which are related to disturbances in plane $x_{1}, x_{3}$. Two of the modes are quasi-longitudinal waves for which the motion of particles of fluid and solid phase take place in or out of phase. The remaining waves are the shear-like waves resulting from the existence of the stiffness of solid phase and viscosity of carrier liquid.

\section{Experimental method}

Measurements of absorption and velocity were carried out using the Matec pulse echo-overlap technique (Fig. 1). The radio-frequency gated amplifier model 755 and gating modulator model 7700 were used to drive the piezo-ceramic transducer. The ultrasonic pulse, on traversal of the sample, was detected by the receiver transducer and amplified in a wide-band amplifier. The resulting pulse-echo train was observed on a CRT display. When the system was properly synchronized by 
the $\mathrm{CW}$ signal from the model 110 high resolution frequency source, the two chosen echoes overlapped on the oscilloscope screen. By tuning the frequency of the CW source, the correct, "cycle-to-cycle", match between the two echoes can be established. Then the velocity of the ultrasonic wave can be evaluated from the expression

$$
v=2 l f,
$$

where $l$ is the distance between the transducers and $f$ - the frequency of the CW source equal to the reciprocal of the round trip time in the sample.

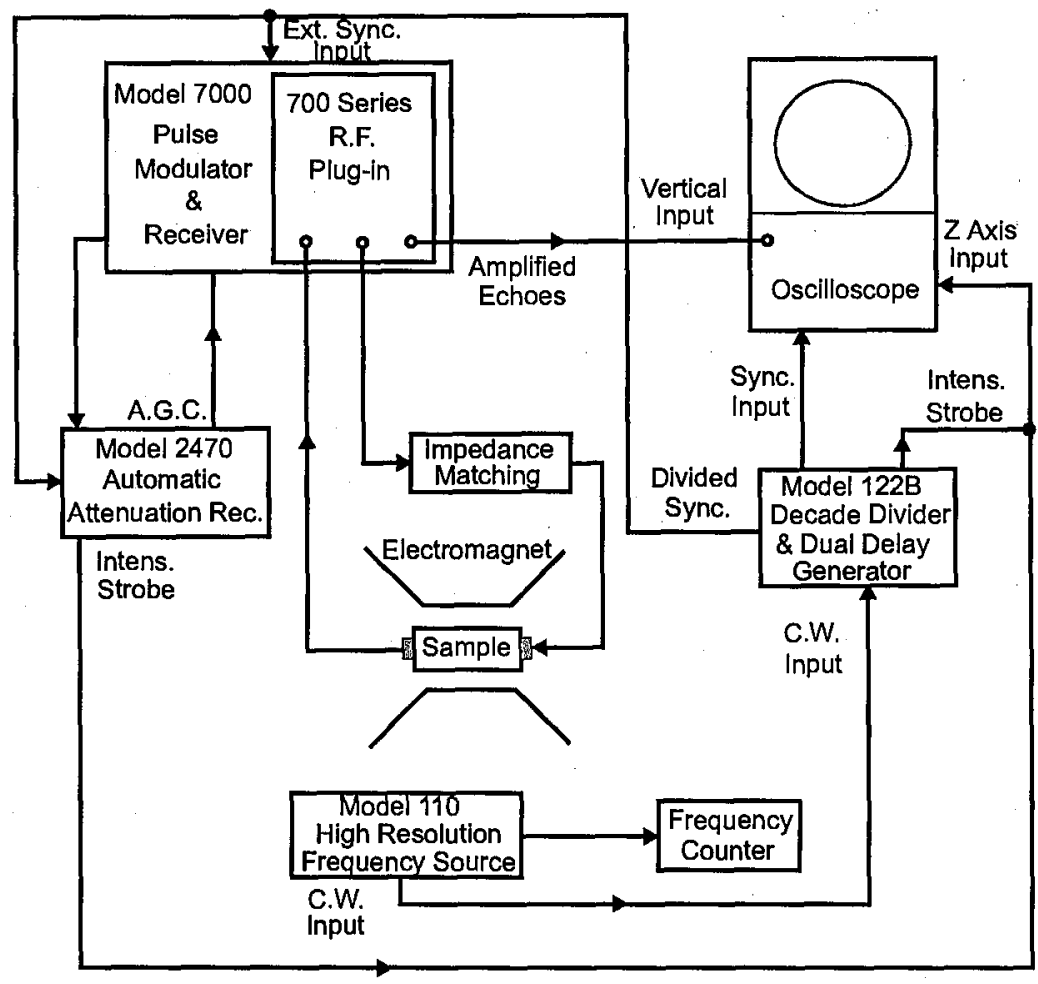

Fig. 1. Block diagram of the experimental setup.

The model 2470B automatic attenuation recorder measures the logarithmic difference (in $\mathrm{dB}$ ) between two selected echoes, say $A_{1}$ and $A_{2}$, if the time gates correctly cover the main portion of the echoes. When $A_{1}$ and $A_{2}$ are two consecutive echoes the $\log \left(A_{1} / A_{2}\right)$ output is proportional to the absorption coefficient of the sample. The variations of $\log \left(A_{1} / A_{2}\right)$, e.g. as a function of the magnetic field strength, give direct changes in the absorption coefficient. The accuracy of our determination of the velocity was of the order of $0.05 \%$ whereas changes in the absorption coefficient were measured with an error less than $1 \%$. The absolute values of the absorption coefficient were accurate within $\pm 5 \%$. A more detailed description of the experimental setup can be found elsewhere [11]. 
In order to measure the effect of the magnetic field on the velocity and attenuation of the ultrasonic wave, the measuring cell (made out of brass) with ferrofluid inside was placed between pole pieces of electromagnet which yielded a maximum field of $1 \mathrm{~T}$ at a spacing of $5 \mathrm{~cm}$. The magnetic field induction was measured to within $0.5 \%$ with a F.W. Bell gaussmeter model 9200 .

\section{Discussion of results}

Predictions of the proposed two-phase model of magnetic liquid with respect to attenuation and wave velocity for variable volume fraction (stiffness) of skeleton are analyzed. The theoretical results are then compared with experimental data for variable strength of magnetic field. The analysis is limited to the waves which propagate along the magnetic field. In such case it is justified to disregard rotational effects of clusters.

The measurements were carried out in a magnetic liquid denoted as EMG-605 (produced by Ferrofluidics Inc.) consisting of magnetite particles $\mathrm{Fe}_{3} \mathrm{O}_{4}$ suspended in water. The values of saturation magnetization, initial susceptibility, volume concentration, and viscosity were $20 \mathrm{mT}, 0.5,3.5 \%$, and $<0.5 \mathrm{~N} \mathrm{~s} \mathrm{~m}^{-2}$ (at $25^{\circ} \mathrm{C}$ ), respectively. The particle distribution was a normal distribution with a maximum at $100 \AA$. The measurements were carried out in $50^{\circ} \mathrm{C}$ at $1.18 \mathrm{MHz}$ and the results are shown in Fig. 2. While the wave velocity increases monotonically with strengthening of magnetic field the attenuation approaches a maximum value and then drops to the level observed for the case of no magnetic field.

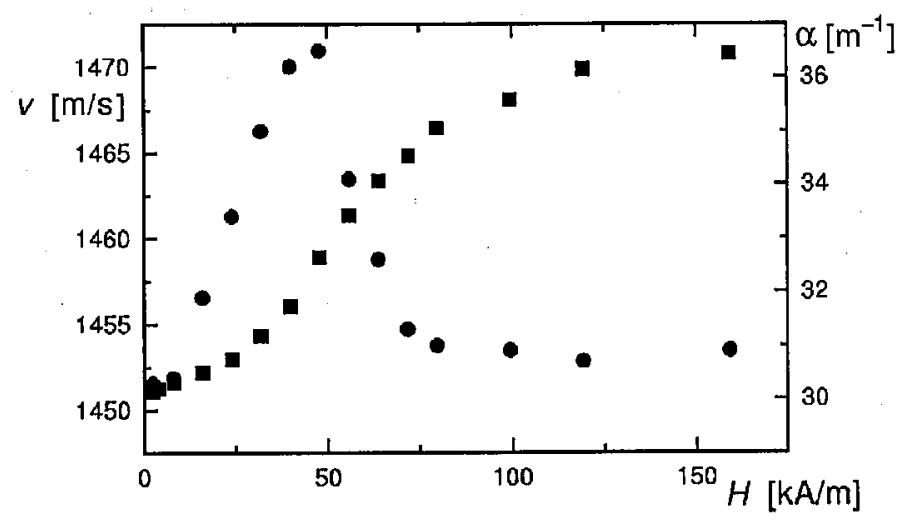

Fig. 2. Velocity and attenuation as a function of magnetic field strength $H$. Data for velocity are denoted by $m$ and for attenuation by $\bullet$. The measurements were carried out in the temperature of $T=50^{\circ} \mathrm{C}$, and for the ultrasonic wave of frequency $f=1.18 \mathrm{MHz}$. The direction of magnetic field was parallel, $\theta=0^{\circ}$, to the ultrasonic wave propagation vector.

In order to obtain numerical predictions of the proposed model a number of assumptions for material parameters must be specified. It is assumed that the increase in magnetic field causes an approximately proportional growth of a number of clusters while the radius of clusters remains constant and equal to $1000 \mathrm{~nm}$. 
For the volume fraction of clusters lower than $\sim 0.02$ (this threshold value was chosen arbitrarily) the interaction of existing clusters is assumed to be negligible and there is no stiff or load bearing skeleton. When the volume fraction increases above the threshold value, clusters start to interact stronger and the interaction is modeled as proportional to the volume fraction of clusters. The interaction takes place solely in direction $x_{3}$. There is no further increase in stiffness of skeleton when all magnetic particles are gathered into clusters, which corresponds to magnetic saturation ( $n$ approaches the maximum value). This pattern of cluster formation is supported by experimental evidences (see e.g. [12]). The assumed dependence of the bulk modulus of skeleton $K_{\mathrm{b}}$ on the volume fraction of clusters with smoothed breaking points is shown in Fig. 3 .

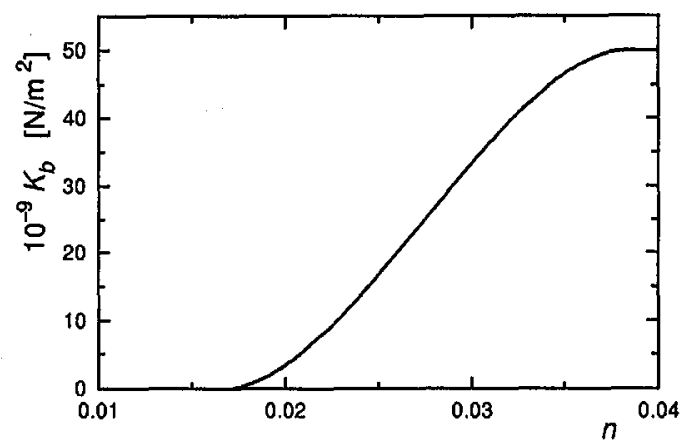

Fig. 3. Dependence of the bulk modulus of skeleton $K_{\mathrm{b}}$ on volume fraction of clusters.

Taking into account the fact that in the case of magnetic liquid the bulk modulus of solid material, $K_{\mathrm{s}}$, is much greater than the bulk modulus of fluid phase, $K_{\mathrm{f}}$, and both parameters are a few orders of magnitude larger than the bulk modulus of skeleton of magnetic liquid, $K_{\mathrm{b}}$, i.e. $K_{\mathrm{s}} \gg K_{\mathrm{f}} \gg K_{\mathrm{b}}$, and using the relationship between macroscopic parameters of the two-phase model of saturated porous media and the moduli of phases, see [13], for a given symmetry of the material one can assume that elastic parameters of the model are as following:

$$
\begin{aligned}
& A=N=L=F=0, \\
& C=K_{\mathrm{f}} \frac{n^{2}}{1-n}+K_{\mathrm{b}}, \\
& R=(1-n) K_{\mathrm{f}}, \\
& M=Q=n K_{\mathrm{f}} .
\end{aligned}
$$

The mass density and bulk modulus of fluid phase are calculated for the series model taking into account the presence of free colloidal particles in fluid. All the coefficients, which determine components of viscous stress tensor in fluid, are assumed to have the same values irrespective to anisotropy and to get the level of the observed attenuation they are taken to be thirteen times larger than the viscosity of carrier fluid $\zeta$. The inertial coupling in the model is disregarded assuming that $c_{t}=c_{n}=0$. The components of the viscous drag are calculated 
assuming that the viscous interaction of a single spherical cluster of radius $r$, moving with velocity $v^{\mathrm{s}}$ in fluid which moves with the velocity $v^{\mathrm{f}}$, is defined by the Stokes formula

$$
F_{\mathrm{S}}=6 \pi \zeta r\left(v^{\mathrm{f}}-v^{\mathrm{s}}\right)
$$

Taking into account that a representation of the Stokes force in the continuum model is obtained by referring the above force to a representative volume of the fluid containing a single cluster $V$ and that the volume fraction of clusters is given by $n=4 \pi r^{3} / 3 V$, the coefficients of viscous coupling are

$$
b_{t}=b_{n}=-\frac{9 n \zeta}{2 r^{2}},
$$

where the negative sign results from the applied notation in Eqs. (1) and (19-20).

In Fig. 4 the predicted by the two-phase model values of attenuation and velocity of the fast quasi-longitudinal wave propagating in $x_{3}$-direction versus the volume fraction of skeleton (clusters) are shown. The exact relation between the volume fraction of skeleton and magnetic field is not yet known. However, there are some evidences [14] that this relation is linear in some range of magnetic field strength. Thus, it seems that the theoretical curve reproduces, at least qualitatively, the behavior of velocity and attenuation of ultrasonic waves in magnetic liquids. The initial increase in attenuation due to a growing amount of suspended in liquid freely moving clusters (clusters which do not interact between themselves) is stopped when clusters start to build up a stiff skeleton. The stiffness of the skeleton limits a relative displacement of clusters with respect to surrounding fluid and this is accompanied by a drop of attenuation with increasing volume fraction of clusters (skeleton stiffness). The stiffness is responsible also for the predicted increase in velocity of the ultrasonic wave.

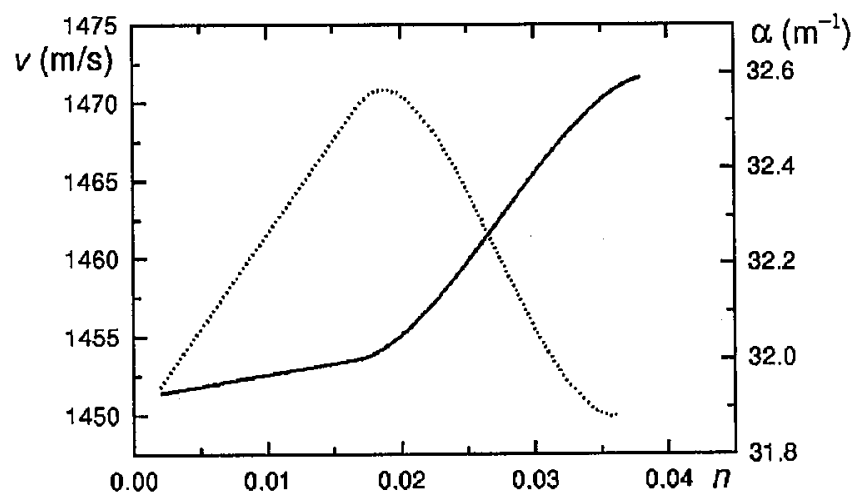

Fig. 4. Predictions of two-phase model for velocity (solid line) and attenuation (dotted line) of ultrasonic waves in magnetic liquid.

Although a further refinement of the model is necessary to include, e.g. changes in viscosity of fluid phase and frequency dependence of interaction between phases, one should notice from the comparison of the results given in Figs. 2 and 4 that there is good qualitative agreement of theoretical results and experimental 
data. It should be noted that the model predicts also the existence of the slow quasi-longitudinal wave. Its velocity - for the presumed material parameters is about ten times smaller, and the attenuation over thousand times larger in comparison with the corresponding parameters for the fast wave. However, with the current measuring setup we were unable to detect this wave experimentally. An analysis of other predictions of the model in relation to the measurements to evaluate, e.g. the effects of anisotropy of the material, and of the role of frequency of ultrasonic waves is necessary and will be approached in the nearest future.

\section{Appendix}

The coefficients appearing in Eqs. (22-27) have the following form:

$$
\begin{aligned}
& D_{11}=(2 N+A) q_{1}^{2}+L q_{3}^{2}+\mathrm{i} \frac{b_{t}}{\omega}-c_{t}-\rho^{\mathrm{s}}, \\
& D_{12}=(F+L) q_{1} q_{3}, \\
& D_{13}=M q_{1}^{2}-\mathrm{i} \cdot \frac{b_{t}}{\omega}+c_{t}, \\
& D_{14}=M q_{1} q_{3} \\
& D_{21}=N q_{1}^{2}+L q_{3}^{2}+\mathrm{i} \frac{b_{t}}{\omega}-c_{t}-\rho^{\mathrm{s}}, \\
& D_{22}=-\mathrm{i} \frac{b_{t}}{\omega}+c_{t}, \\
& D_{31}=(F+L) q_{1} q_{3}, \\
& D_{32}=C q_{3}^{2}+L q_{1}^{2}+\mathrm{i} \frac{b_{n}}{\omega}-c_{n}-\rho^{\mathrm{s}}, \\
& D_{33}=Q q_{1} q_{3}, \\
& D_{34}=Q q_{3}^{2}-\mathrm{i} \frac{b_{n}}{\omega}+c_{n}, \\
& D_{41}=M q_{1}^{2}-\mathrm{i} \frac{b_{t}}{\omega}+c_{t}, \\
& D_{42}=Q q_{1} q_{3} \\
& D_{43}=R q_{1}^{2}+\mathrm{i} \omega(2 \mu+\alpha) q_{1}^{2}+\mathrm{i} \omega \delta q_{3}^{2}+\mathrm{i} \frac{b_{t}}{\omega}-c_{t}-\rho^{\mathrm{f}}, \\
& D_{44}=R q_{1} q_{3}+\mathrm{i} \omega \beta q_{1} q_{3}+\mathrm{i} \omega \delta q_{1} q_{3}, \\
& D_{51}=-\mathrm{i} \frac{b_{t}}{\omega}+c_{n}, \\
& D_{52}=\mathrm{i} \omega \mu q_{1}^{2}+\mathrm{i} \omega \delta q_{3}^{2}++\mathrm{i} \frac{b_{t}}{\omega}-c_{t}-\rho^{\mathrm{f}}, \\
& D_{61}=M q_{1} q_{3}, \\
& D_{62}=Q q_{3}^{2}-\mathrm{i} \frac{b_{n}}{\omega}-c_{n}, \\
& D_{63}=R q_{1} q_{3}+\mathrm{i} \omega \beta q_{1} q_{3}+\mathrm{i} \omega \delta q_{1} q_{3}, \\
& D_{64}=R q_{3}^{2}+\mathrm{i} \omega(\gamma+\beta) q_{3}^{2}+\mathrm{i} \omega \delta q_{1}^{2}+\mathrm{i} \frac{b_{n}}{\omega}-c_{t}-\rho^{\mathrm{f}} .
\end{aligned}
$$




\section{Acknowledgment}

Supported by the Committee for Scientific Research grant No. 2P03B 07912.

\section{References}

[1] I. Nakatani, in: Proc. Int. Symp. on Hydrodynamics of Magnetic Liquids and its Application, Sendai, 1997, p. 9.

[2] I.E. Tarapov, Magnetohydrodynamics 1, 1 (1972).

[3] J.D. Parsons, J. Phys. D 8, 1219 (1975).

[4] S. Taketomi, Jpn. J. Appl. Phys. 22, 1137 (1983).

[5] A.I. Lipkin, Magn. Gidrodin. No. 3, 25 (1985).

[6] W.W. Gogosow, W.W. Martynow, S.I. Curikow, G.A. Szaposznikowa, Magn. Gidrodin. No. 2, 19 (1987).

[7] R. Bădescu, E. Luca, V. Bădescu, N. Rezlescu, J. Magn. Magn. Mater. 170, 235 (1997).

[8] R.M. Bowen, in: Theory of Mixtures, Ed. A.C. Erring, in series Continuum Physics, Vol. 3, Academic Press, New York 1976, p. 1.

[9] M.D. Sharma, M.L. Gogna, J. Acoust. Soc. Am. 90, 1068 (1991).

[10] M. Kaczmarek, J. Kubik, Arch. Mech. 41, 769 (1989).

[11] A. Skumiel, M. Łabowski, T. Hornowski, J. Phys. D 30, 25 (1996).

[12] J. Černák, P. Macko, J. Magn. Magn. Mater. 123, 107 (1983).

[13] M.A. Biot, D.G. Willis, J. Appl. Mech. 24, 594 (1957).

[14] A. Skumiel, T. Hornowski, Acoust. Lett. 20, 121 (1996). 